\title{
Impact of Next Generation Cognitive Radio Network on the Wireless Green Eco system through Signal and Interference Level based K Coverage Probability
}

\author{
Er. A.S.Kang ${ }^{\star}$, Renu Vig \\ UIET, Panjab University Chandigarh, India \\ e-mail: askang_85@yahoo.co.in
}

\begin{abstract}
Land mobile communication is burdened with typical propagation constraints due to the channel characteristics in radio systems. Also, the propagation characteristics vary form place to place and also as the mobile unit moves, from time to time. Hence, the tramsmission path between transmitter and receiver varies from simple direct LOS to the one which is severely obstructed by buildings, foliage and terrain. Multipath propagation and shadow fading effects affect the signal strength of an arbitrary TransmitterReceiver due to the rapid fluctuations in the phase and amplitude of signal which also determines the average power over an area of tens or hundreds of meters. Shadowing introduces additional fluctuations, so the received local mean power varies around the area-mean. The present paper deals with the performance analysis of impact of next generation wireless cognitive radio network on wireless green eco system through signal and interference level based $k$ coverage probability under the shadow fading effects.
\end{abstract}

Keywords: radio network, wireless cognitive radio network, signal.

\section{Introduction}

Wireless sensor networks (WSNs) consist of a large number of densely deployed sensor nodes to monitor and track phenomenon or objects of interest in a specified region. Sensor networks pose a number of challenging conceptual and optimization problems such as localization,deployment and tracking [1-2]. One of the fundamental problems in wireless sensor networks relates to coverage which refers to how well sensors monitor or track the events.In general, coverage can be considered as the measure of quality of service in a sensor network. The coverage requirements may change after a network has been deployed due to changes in the requirements of an application or environmental conditions[3].Sensing coverage problem has been expressed as area coverage and point coverage. Different applications require different degrees of sensing coverage; for example, some applications may require that a location in a region be monitored by only one sensor, while other applications require significantly higher number of sensors for the same [4].The most general way of defining sensing coverage is to consider the ratio of sensing area to the area of interest, which means the fraction of area that can be covered by the sensor network. The achievable sensing coverage strongly depends on the node deployment planning. However, sensors should be deployed in such a way that it should maximize the coverage and minimize the required number of sensors.Planned node deployment is not always possible in some unstructured sensor network applications like battlefield surveillance, forest monitoring, and so forth.Therefore, in such applications, sensor nodes are randomly deployed to provide the required coverage.However, it may be important to predict the minimum number of sensor nodes that provide an acceptable level of coverage.Many of the sensor networks concentrate on detecting specific signals such as thermal energy, acoustic signal, seismic signal,radio waves, light waves or magnetic field, in the preferred sensing area [5]. 


\section{Path-loss law}

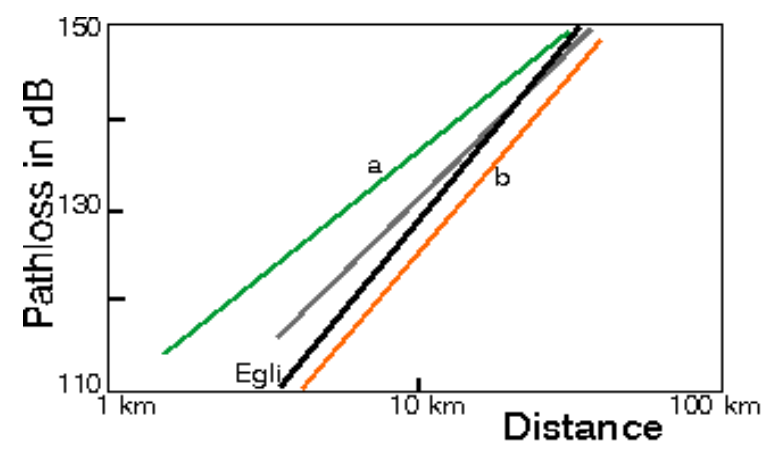

Figure 1. 99 Average path loss versus distance in UHF bands as measured in Northern Germany. (a, green): forest terrain; (b, orange): open area;

(grey): average of (a) and (b); (black): Egli's model[6]

These signals are affected by environmental factors like noise, interference, reflection of signals, obstructions in the propagation path, and movement of other objects. Sensing signals have extra power loss due to environmental factors besides path loss. This extra power loss results into large deviations in the received signal strength. The deviation in the received signal strength due to obstructions in propagation path is known as shadowing whereas deviation due to reflections is known as multipath fading. The shadowing and multipath fading phenomena adversely affect the quality sensing coverage [6].

\section{Implications for Cognitive Radio Network Planning}

If one extends the distinction between large-area and small-area shadowing, the definition of shadowing covers any statistical fluctuation of the received local-mean power about a certain area-mean power, with the latter determined by predictable large-scale mechanisms. Multipath propagation is separated from shadow fluctuations by considering the local-mean powers. That is, the standard deviation of the shadowing will depend on the geographical resolution of the estimate of the area-mean power. A propagation model which ignores specific terrain data produces about $12 \mathrm{~dB}$ of shadowing. On the other hand, prediction methods using topographical data bases with unlimited resolution can, at least in theory, achieve a standard deviation of $0 \mathrm{~dB}$. Thus, the standard deviation is a measure of the impreciseness of the terrain description. If, for generic system studies, the large-scale path loss is taken of simple form depending only on distance but not on details of the path profile, the standard deviation will necessarily be large. On the other hand, for the planning of a practical network in a certain known environment, the accuracy of the large-scale propagation model may be refined. This may allow a spectrally more efficient planning if the cognitive-cellular layout is optimized for the propagation environment. With shadowing, the interference power accumulates more rapidly than proportionally with the number of signals. The accumulation of multiple signals with shadowing is a relevant issue in the planning of cellular networks[7-8].

\section{5G Wireless Radio Network: Next Wave of Digital Society}

The use of mobile communication networks has increased significantly in the past decades, in terms of complexity of applications, their required capacities, and heterogeneity of device types. So far, this trend has always been met by significant technological advancements and will continue to increase. By 2020,Europe has to pave the way for a new generation of converged wired and wireless communication networks, which has to be developed and deployed to move forward to a future networked society. Here,we present our perspective on such a $5 G$ wireless cognitive radio access network and focus especially on the arising challenges and new technologies that enable us to meet these challenges. Looking back at the development of $3 G$ (UMTS,HSPA) and 4G (LTE,LTE Advanced) it is clear that these

IJEEI Vol. 5, No. 1, March 2017: $69-76$ 
generations of mobile networks focused on creating new physical radio transmission schemes in order to meet new capacity requirements. From our point of view,5G networks should consider both wireless and wired parts targeting a fully integrated solution. Furthermore, in order to address the user oriented challenges, we foresee a continued evolution of the existing functions, e.g.network densification into ultradense networks and device to device communications, as well as development of new functions such as moving(mobile) networks and massive machine communications.5th generation mobile networks or wireless systems denote the next major phase of mobile telecommunications standards beyond the current 4G/IMT-Advanced standards. The Next Generation Mobile Networks Alliance defines 5G network requirements as Data rates of several tens of $\mathrm{Mb} / \mathrm{s}$ should be supported for tens of thousands of users, $1 \mathrm{Gbit} / \mathrm{s}$ to be offered, simultaneously to tens of workers on the same office floor, Several hundreds of thousands of simultaneous connections to be supported for massive sensor deployments, Spectral efficiency should be significantly enhanced compared to 4G.Coverage should be improved, Signaling efficiency could be enhanced[9]. Hence,5G will prove to be key wireless technology for the next generation cognitive radio communication networks. Figureure5.100 shows the proposed wireless cognitive cellular radio network to check the congested RF spectrum.

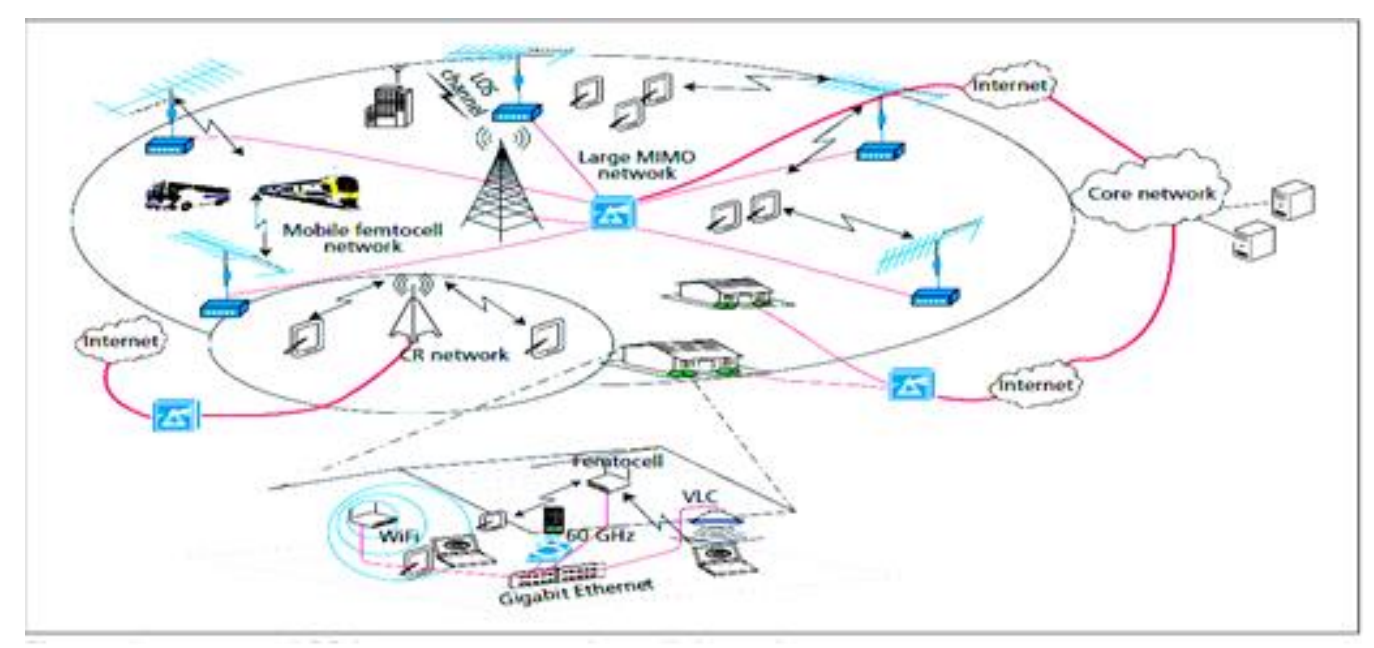

Figure 2.100 Proposed 5G Wireless Cognitive Radio Network[10].

\section{Interference Management for CR Networks}

A major issue in interference-tolerant $\mathrm{CR}$ networks in $5 \mathrm{G}$ is how to reliably and practically manage the mutual interference of $C R$ and primary systems. Regulating the transmit power is essential for the CR system to coexist with other licensed systems. An interference temperature model is introduced for this purpose to characterize the interference from the CR to the licensed networks. Interference cancellation techniques should also be applied to mitigate the interference at $\mathrm{CR}$ receivers. Another issue in interference-tolerant CR networks is that a feedback mechanism is important to periodically inform the CR network about the current interference status at the licensed system. A practical solution is that the interference state information can be sent from licensed systems and collected by a central unit (or a third party system).Any CR network should first register to the central unit in order to be updated regarding the allowed spectrum and interference. Alternatively, the CR transmitters can listen to beacon signals transmitted from the primary receivers and rely on the channel reciprocity to estimate the channel coefficient. In this case, the CR transmitters can cooperate among themselves to regulate the transmit power and prevent the interference at the primary receivers being above the threshold [11]. 


\section{Coverage Probability Estimation under Shadow Fading Effect: Methodology}

The coverage probability is the probability that such a ratio is above a given threshold. In particular, we are interested in the probability that there is some antenna above threshold, which provides a certain "gain": if fading increases the Ec of some antenna, that not only reduces the chance that other antennas are above threshold, by increasing 10, but it also, of course, increases the chance that the given antenna is above threshold. We will derive an expression for coverage probability that conservatively accounts for such gain. We can estimate the coverage probability[12] that a location is uncovered, so that $(\mathrm{l} / \mathrm{Ek})>\mathrm{tkfor}$ all $\mathrm{k}$. Also,

$\mathrm{I}=\eta+\sum_{\mathrm{j}} \mathrm{E}_{\mathrm{j}}$

Assuming that $t_{k}=t$, the desired probability is equal to

$\prod \operatorname{Prob}\left\{\mathrm{l}>\mathrm{tE}_{\mathrm{k}} \quad \mathrm{I}>\mathrm{t}_{\max } \mathrm{E}_{\mathrm{j}}(\mathrm{j}<\mathrm{k})\right.$

Let $I_{k}=\eta+\sum E_{j}(j>k)$. The condition for given $k$ implies that

$\mathrm{t} \mathrm{l}=\mathrm{t} \sum \mathrm{E}_{\mathrm{j}}+\mathrm{t} \mathrm{E}_{\mathrm{k}}+\mathrm{t} \mathrm{I}_{\mathrm{k}}<(\mathrm{k}-1) \mathrm{I}+\mathrm{t} \mathrm{E}_{\mathrm{k}}+\mathrm{t} \mathrm{I}_{\mathrm{k}}$. so that $\mathrm{I}<[\mathrm{t} /(\mathrm{t}-\mathrm{k}+1)]\left(\mathrm{E}_{\mathrm{k}}+\mathrm{I}_{\mathrm{k}}\right)$

Further, we can use the estimate probability as

$$
\begin{aligned}
& \operatorname{Prob}\left\{l>t E_{k} \quad I>t_{\max } E_{j}(j<k)\right. \\
& <\operatorname{Prob}\left\{[t /(t-k+1)]\left(E_{k}+I_{k}\right)>t E_{k}, I>t_{\max } E_{j}(j<k)\right\} \\
& \approx \operatorname{Prob}\left\{[t /(t-k+1)]\left(E_{k}+I_{k}\right)>t E_{k}\right. \\
& =\operatorname{Prob}\left\{\left(I_{k} / E_{k}\right)>t-k\right\}
\end{aligned}
$$

\section{Simulation Results and Discussion}

Here we have calculated and plotted SINR-based probability of k-coverage via simulation of model and integration method and compared our results with those obtained by H.P. Keeler, Inria Paris/ENS,2013[13]. Here the performance parameters which have been taken under consideration are base station density represented by lambda $=0.2887 / 2$; In this comparatative Simulation Study, K and betaConst values correspond to Walfisch-lkegami model for a urban environment where betaConst $=4.2$ is nothing but path-loss exponent and $K=6910$. The log normal parameters which have been used are sigmDb=10; sigma $=\operatorname{sigmDb} / 10^{*} \log (10)$; ESTwoBeta=exp(sigma^2* $\left(2\right.$-betaConst)/betaConst $\left.{ }^{\wedge} 2\right)$; The model constant incorporates the model parameters as stated in equation below.

\section{$\mathrm{a}=$ lambda ${ }^{\star} \mathrm{pi}^{*} \mathrm{EST}$ woBeta/K^2;}

While the noise parameters are helpful in calculating $W \cdot N=10^{\wedge}(-96 / 10) / 1000$; $\mathrm{P}=10^{\wedge}(62.2 / 10) / 1000 ; \mathrm{W}=\mathrm{N} / \mathrm{P} ;$ In this entire process, the SINR threshold values which have been taken are as follows

$$
\text { tMinDb=-10;tMaxDb=25;tValuesDb=(tMinDb:tMaxDb)'; }
$$

(values in $\mathrm{dB}$ ), $\mathrm{tValues}=10 .^{\wedge}$ (tValuesDb/10); tNumb=length(tValues); while the coverage number has been varied from 1 to $2 . k=1$; To achieve the analytic/integration results, we have made the usage of following function.

numbMC=10^3;PCov=funProbCov(tValues, betaConst, $\mathrm{W}^{*} \mathrm{a}^{\wedge}$ (-betaConst/2), numbMC,k); Ultimately, the Simulation section shows the results which are there obtainable after incorporating fading environment effect. simNumb $=10^{\wedge} 4 \%$ number of simulations; diskRadius=20; PcovSim = funSimLogNormProbCov (tValues, betaConst, K, lambda, sigma, $\mathrm{W}$, diskRadius, simNumb, $k$ ); The results are plotted by using the commands namely $\mathrm{Pn}=1-\mathrm{Pcov}$; PnSim=1-PCovSim; plot(tValuesDb, PnSim, 'o', tValuesDb, Pn); grid; legend (['Simulation',legendLabel], ['Integration', legendLabel], xlabel('T(dB)'); ylabel('1-P_c(T)') 


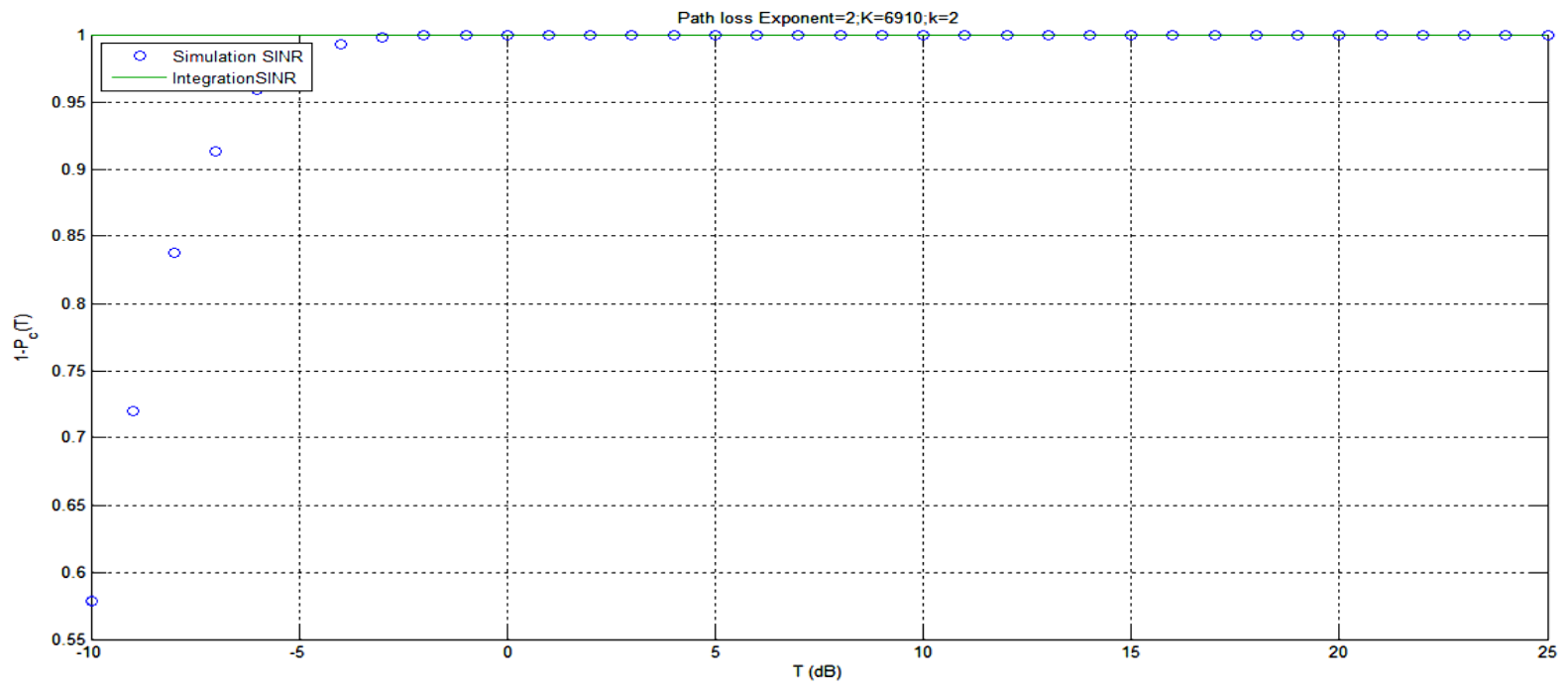

Figure 3.101. 1- $\mathrm{Pc}(\mathrm{T})$ versus $\mathrm{T}(\mathrm{dB})$ Simulation and Integration Method;Path Loss Exponent $=2 ; \mathrm{K}=6910 ;$ Coverage number $\mathrm{k}=2$.

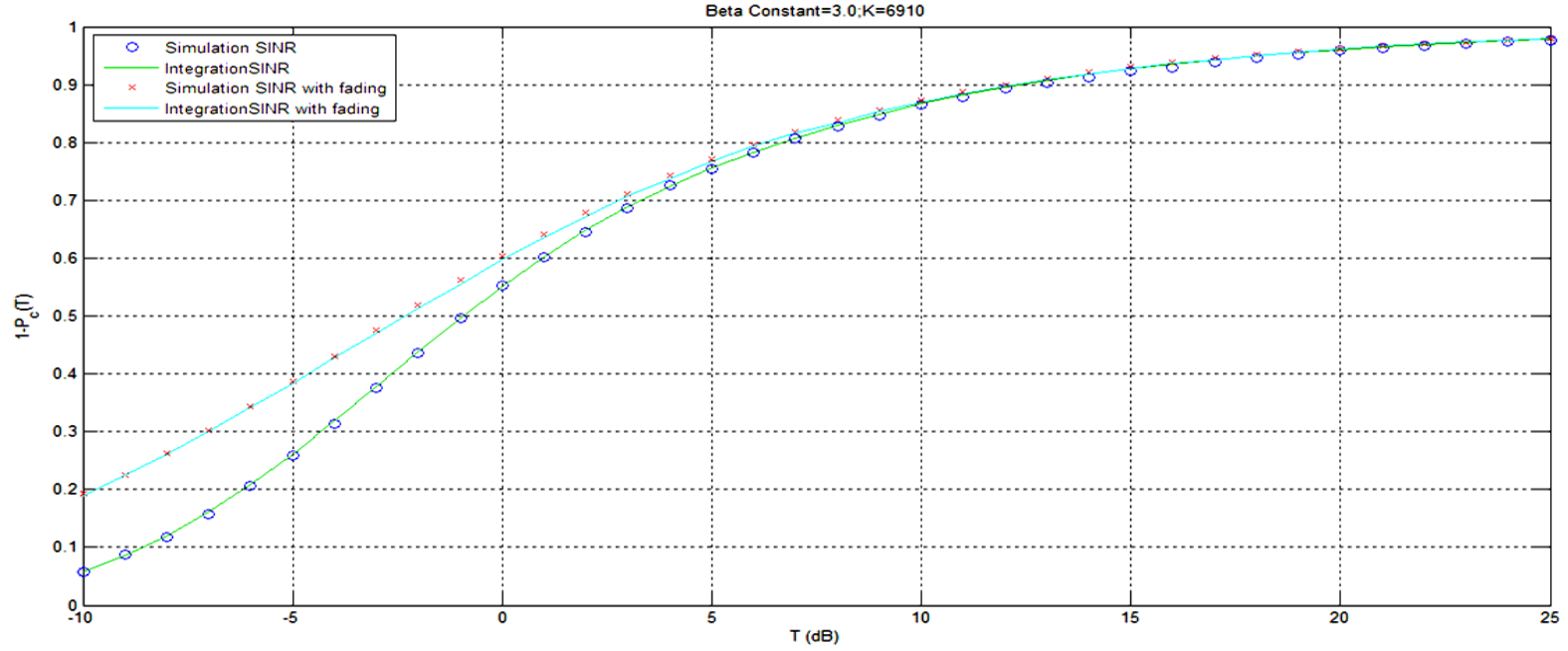

Figure 4.102.1- $\mathrm{Pc}(\mathrm{T})$ versus $\mathrm{T}(\mathrm{dB})$ Simulation and Integration Method;Path Loss Exponent $=3.0 ; \mathrm{K}=6910$; Coverage number $\mathrm{k}=1$. 


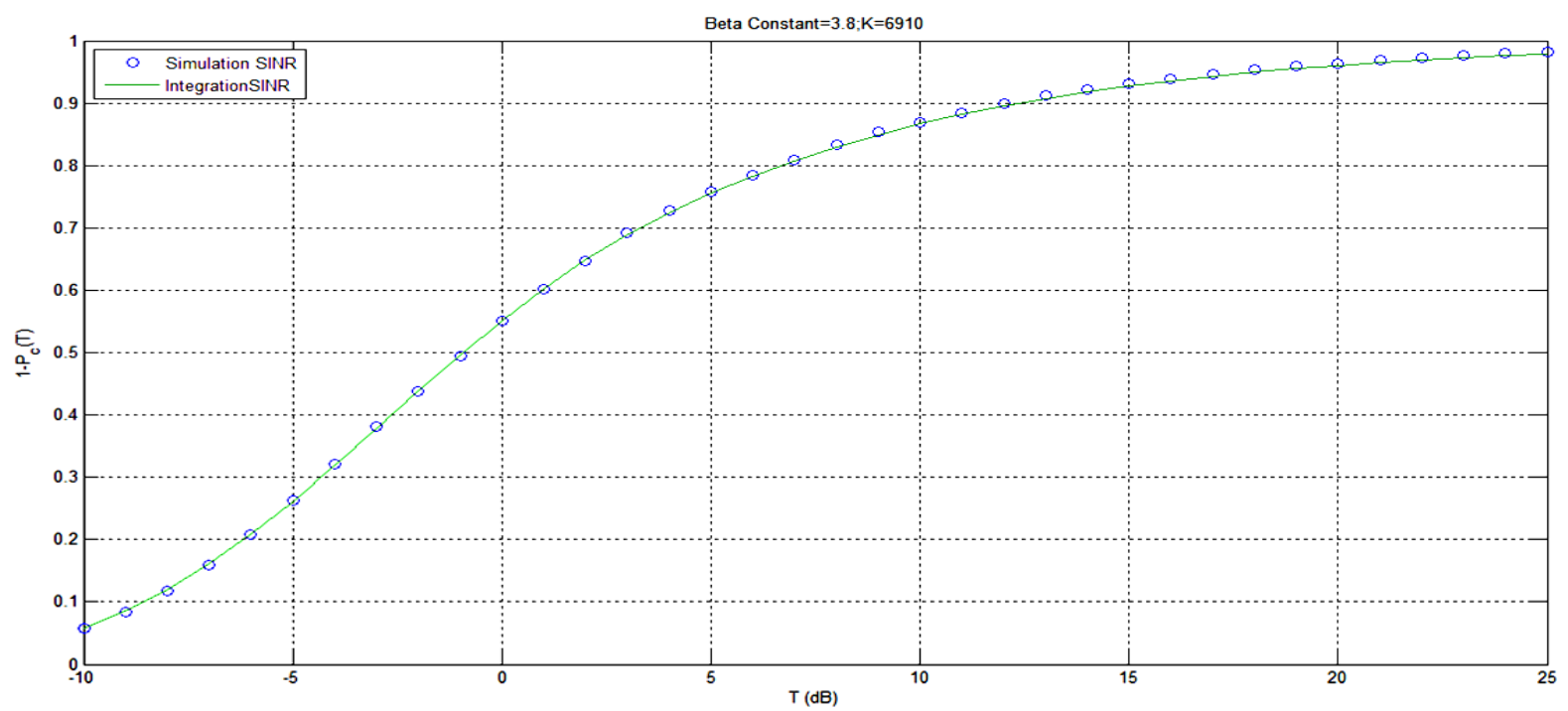

Figure 5.103.1- $\mathrm{Pc}(\mathrm{T})$ versus $\mathrm{T}(\mathrm{dB})$ Simulation and Integration Method;Path Loss Exponent $=3.8 ; \mathrm{K}=6910 ;$ Coverage number $\mathrm{k}=1$

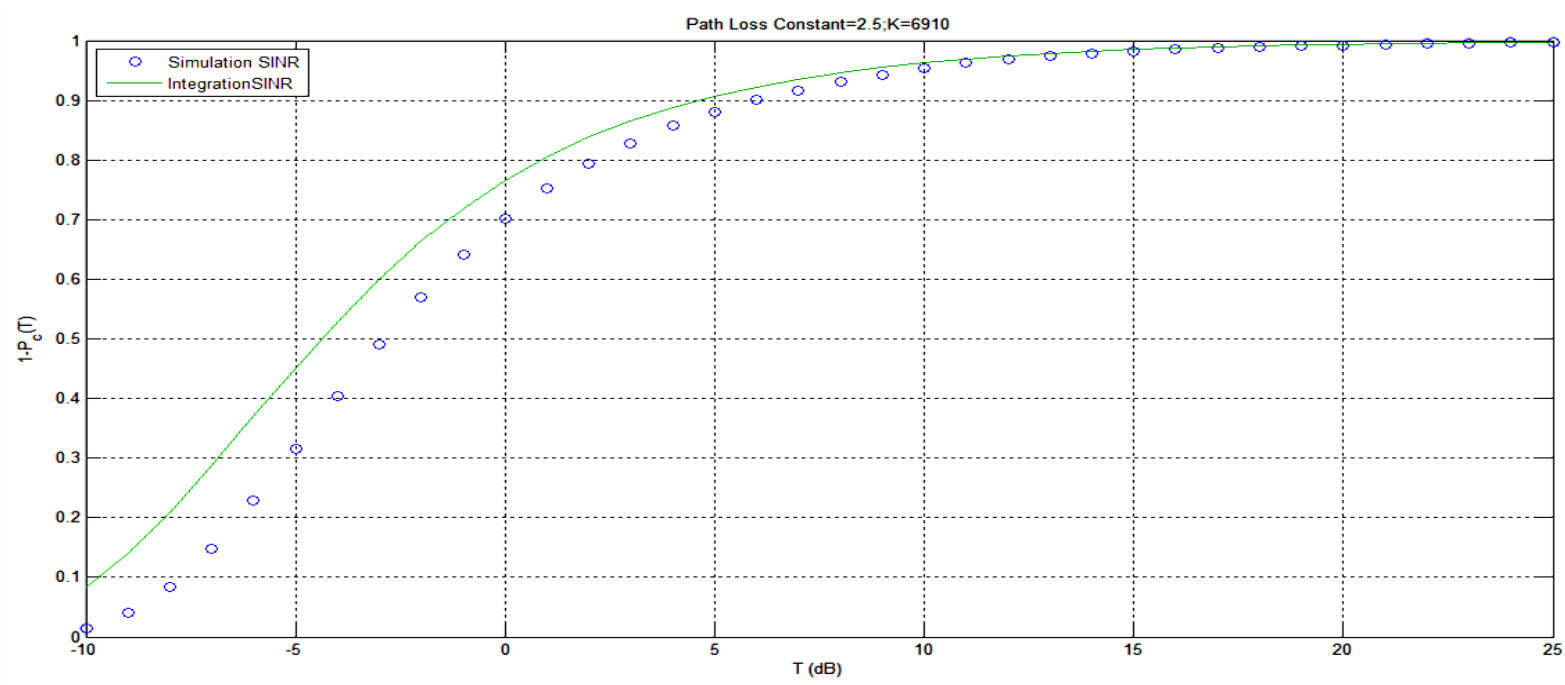

Figure 6.104.1- $\mathrm{Pc}(\mathrm{T})$ versus $\mathrm{T}(\mathrm{dB})$ Simulation and Integration Method;Path Loss Exponent $=2.5 ; \mathrm{K}=6910 ;$ Coverage number $\mathrm{k}=1$ 


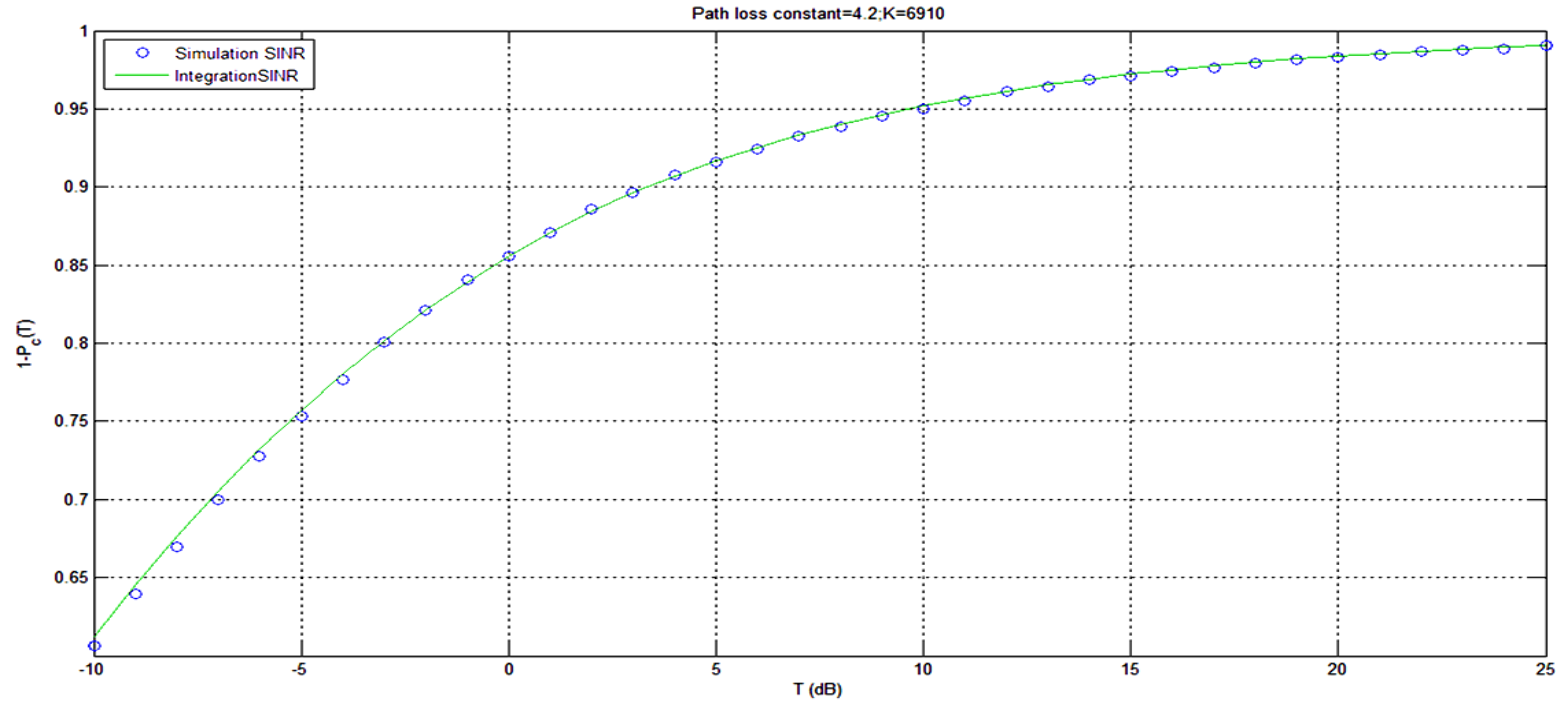

Figure 7.105.1- $\mathrm{Pc}(\mathrm{T})$ versus $\mathrm{T}(\mathrm{dB})$ Simulation and Integration Method;Path Loss Exponent $=4.2 ; \mathrm{K}=6910 ;$ Coverage number $\mathrm{k}=1$

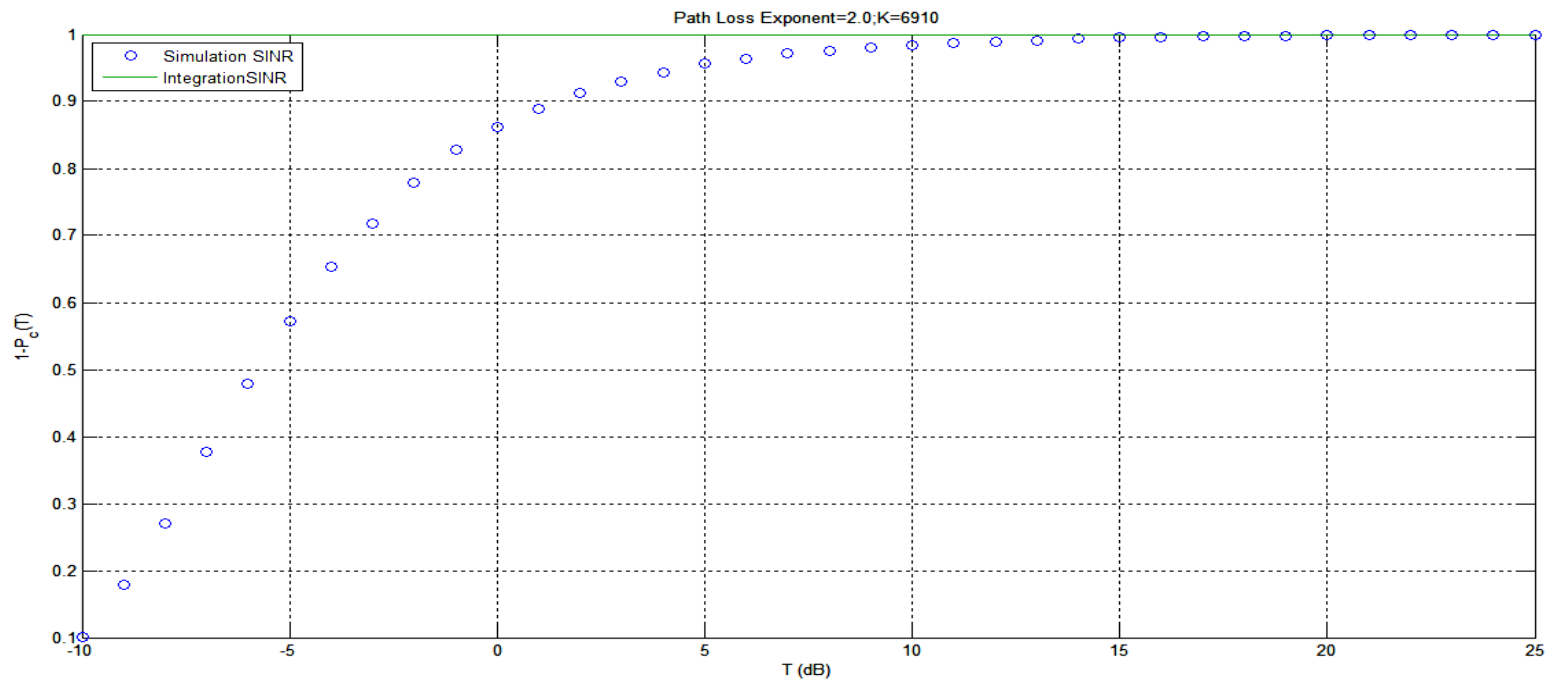

Figure 8.106.1- $\mathrm{Pc}(\mathrm{T})$ versus $\mathrm{T}(\mathrm{dB})$ Simulation and Integration Method;Path Loss Exponent=2.0; $\mathrm{K}=6910 ;$ Coverage number $\mathrm{k}=1$

Table 1. Comparatative Analysis of Walfisch Ikegami model with its Modified Variant(Proposed Model) under Urban Environmet .

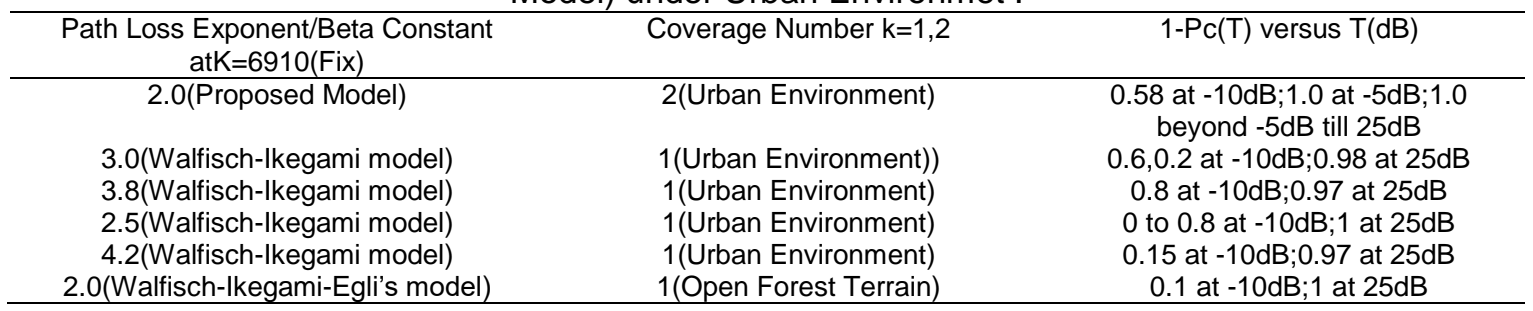




\section{Conclusion}

All the tabulated results have been presented after their validation and comparatative analysis with modified Walfisch-lkegami model variants at fix $\mathrm{K}=6910$ but variable coverage probability number $\mathrm{k}(1,2)$ Beyond $\mathrm{k}=2$ the trend in results changes drastically with 1$\mathrm{Pc}(\mathrm{T})=0.75$ (Constant) from $-10 \mathrm{db}$ to $25 \mathrm{~dB}$ (Threshold). $5 \mathrm{G}$ Cognitive Radio Network is an innovative SDR technique which has been considered as one of the promising technologies to improve the utilization of congested RF Spectrum.Adopting CR is motivated by the fact that a large portion of spectrum is underutilized most of the time[14-16].

\section{References}

[1] Akyildiz IF, Su W, Sankarasubramaniam Y, Cayirci E. A survey on sensor networks. IEEE Communications Magazine. 2002; 40(8): 102-114.

[2] Hossain A, Biswas PK, Chakrabarti S. Sensing models and its impact on network coverage in wireless sensor network. Proceedings of the IEEE Region 10 Colloquium and 3rd International Conference on Industrial and Information Systems (ICIIS '08); India. 2008: 1-5.

[3] Zaho F, Guibas L. Wireless Sensor Networks: An Information Processing Approach. Elsevier. 2004.

[4] Bereketli A, Akan OB. Communication coverage in wireless passive sensor networks. IEEE Communications Letters. 2009; 13(2): 133-135.

[5] Mao G, Anderson BDO, Fidan B. Path loss exponent estimation for wireless sensor network localization. Computer Networks. 2007; 51(10): 2467-2483.

[6] Zhu C, Zheng C, Shu L, Han G. A survey on coverage and connectivity issues in wireless sensor networks. Journal of Network and Computer Applications. 2012; 35(2): 619-632.

[7] Wang X, Xing G, Zhang Y, Lu C, Pless R, Gill C. Integrated coverage and connectivity configuration in wireless sensor networks. Proceedings of the 1st International Conference on Embedded Networked Sensor Systems (SenSys '03). New York. 2003: 28-39.

[8] Xing G, Wang X, Zhang Y, Lu C, Pless R, Gill C. Integrated coverage and connectivity configuration for energy conservation in sensor networks. ACM Transactions on Sensor Networks. 2005; 1(1): 3672.

[9] 5G White Paper - Executive Edition by NGMN Alliance.

[10] Cheng-Xiang Wang, Fourat Haider et al. Cellular Architecture and Key Technologies for 5G Wireless Communication Networks. IEEE Communications Magazine. 2014: 122-130.

[11] Kenneth L.Clarkson, John D.Hobby. A Model of Coverage Probability under Shadow Fading. IEEE Transactions on Communication. 2003: 11.

[12] www.googlescholar.com.

[13] HP Keeler and Inria. Paris,2013. Walfisch-lkegami Model. www.mathworks.com

[14] AS Kang. Analysis of Noisy Fading Channel under strategic conditions. Proc. International Conference on Next Generation \& Computing. Institution of Engineers, Technocrats, Academician. Network. 2010: 330-335.

[15] AS Kang, Renu Vig. Simulation Analysis of Modified Filter Bank Multicarrier for Physical Layer Cognitive Radio under Radio Environment. Invertis Journal of Science and Technology. 2014; 7(4): 214.

[16] AS Kang, Renu Vig. Computer Aided BER Performance Analysis of FBMC Cognitive Radio for Physical Layer under the Effect of Binary Symmetric Radio Fading Channel. SPRINGER-Journal of Wireless Personal Communications. 2015; 81(2): 15.

IJEEI Vol. 5, No. 1, March 2017: 69-76 